\title{
Study of Combining Ability Analysis in Barley (Hordeum vulgare L.)
}

\author{
Deepak Panwar* and Hemlata Sharma \\ Department of Genetics and Plant Breeding, Rajasthan College of Agriculture, \\ MPUAT, Udaipur (Rajasthan)-313001, India
}

*Corresponding author

\begin{abstract}
A B S T R A C T
9 diverse barley genotypes were selected and crossed in a diallel (without reciprocals) mating design to evaluate combining ability effects to identify promising crosses for 13 quantitative characters including yield and its components. Analysis of variance for diallel revealed highly significant differences for most of the traits investigated. Parent BH 393 has been excellent general combiner for majority of traits viz., days to 50 per cent heading,

Keywords Barley, Combining ability, Diallel, GCA, SCA

\section{Article Info}

Accepted: 04 November 2019 Available Online: 10 December 2019 days to 75 per cent maturity. Another parent BH 959 has good general combiner for plant height, flag leaf area, number of effective tillers per plant, spike length, number of grains per spike, number of spikelets per plant, 1000-grain weight, and grain yield per plant. Followed by Parent RD 2786 has been good general combiner for protein content. Parents RD 2715, BH 959, BH 393 can be utilizing for developing of a variety by exploiting additive gene action. BH 959 and DWRB 64 these both the parents are of higher yielding these both the parent of higher grain yielding crosses viz., RD $2786 \times \mathrm{BH} 959$, RD $2552 \times$ DWRB 64, and RD $2035 \times$ RD 2786. A perusal of specific combining ability effects revealed that positive significant sca effects for grain yield per plant was observed in eleven crosses viz., DWRB $91 \times$ BH 959, RD $2552 \times$ DWRB 64, RD $2035 \times$ RD 2786, RD $2035 \times$ BH 393, DWRB $92 \times$ BH 393 RD $2715 \times$ DWRB 64, RD $2715 \times$ DWRB 91, BH $959 \times$ DWRB 64, RD $2552 \times$ RD 2715, RD $2035 \times$ DWRB 92 and DWRB $91 \times$ DWRB 64 expressed higher positive significant sca effects ranged from 1.17 to 2.50 along with good per se performance from 14.44 to 21.07 . It is, therefore, the selective parents and crosses could be utilized for developing desirable crosses with high yielding towards exploiting the hybrid vigor or other related traits under crop improvement.
\end{abstract}

\section{Introduction}

Barley (Hordeum vulgare L., $2 \mathrm{n}=2 \mathrm{x}=14$ ) is the world's fourth most important cereal crop after Wheat (Triticum aestivum L.), Maize (Zea mays L.) and Rice (Oryza sativa L.). It belongs to family Poaceae. Barley is popularly known as "Jau" in Hindi and one of the most important cereal grain crops in India. It is cultivated as a rabi season crop in India and sowing is done from Oct. to Dec. \& harvesting from March to May. Barley is most paramount cereal crop and considered as the first cereal domesticated for use by man as food and feed (Potla et al., 2013). This crop requires temperature of $12^{\circ} \mathrm{C}$ to $16^{\circ} \mathrm{C}$ at growing stage and about $30^{\circ} \mathrm{C}$ to $32^{\circ} \mathrm{C}$ at maturity. It is very susceptible to frost at any stage of its growth 
and has very good tolerance to drought conditions. It is grown under marginal to sub marginal land and mostly under rained condition. It is an essential crop of North India and among three spp. of barley Viz., Two-row barley $(H$. distichum), four-row barley $(H$. tetrastichum), six-row barley (H. vulgare) and husk and huskless barley are available. It is an economically important crop plant, the fourth cereal worldwide in terms of the planting area, utilized almost 60 per cent as animal feed, around 30 per cent for malt production, 7 per cent for seed production and only 3 per cent for human food (Baik et al., 2008). It is produced primarily as animal feed, while it has same nutritive value as corn. It is graining importance in neutraceutical diets and has potential health benefit due to soluble fibre Bglucan which helps in lowering cholesterol level, improving lipid metabolism (Koulagi et al., 2018). It contains high amounts of carbohydrates, moderate amounts of protein, calcium and phosphorus and it has small amounts of the vitamin B. By-products of the brewing process and malt sprouts are also used as livestock feed. Sometimes barley is grown as a hay crop in some areas and only the smooth varieties or awn less variety are used in hay production. Winter barley also can be used for hay if pasteurized before the stems start to elongate. Barley is also used in liquor industry and breakfast foods.

Since per se performance of parents may not reveal their combining ability, so the information on nature of gene actions and their expression in terms of combining ability is necessary. (Patial et al., 2016). The main objectives of this study were to identify of superior parents and crosses combinations from half diallel mating design to obtained high grain yield and better malting quality grains in barley.

The combining ability provide information of the nature of gene action involved in the inheritance of various characters and there by breeding methodology to be used. The nature of gene action would help in predicting the efficiency of selection in population. A distinct type of gene action, its magnitude and constitution of genetic architecture are of fundamental importance to plant breeder.

\section{Materials and Methods}

For the present experiment 9 parents were crossed in diallel (without reciprocals) mating design to obtain 36 crosses during rabi 201718. The experimental material thus consisted of 9 parents 36 crosses and two checks viz., RD 2899 and BH 946. Nine genetically diverse parents namely RD 2035, RD 2552, RD 2786, RD 2715, DWRB 91, BH 959, DWRB 92, DWRB 64, and BH 393. Experiment was evaluated in randomized block design (RBD) in 3 replications. The experiment carried out at the research farm, Rajasthan College of Agriculture, Maharana Pratap University of Agriculture and Technology, Udaipur (Rajasthan) during rabi 2018-2019. The observations recorded for thirteen characters viz., days to 50 per cent heading, days to 75 per cent maturity, plant height, flag leaf area, number of effective tillers per plant, spike length, number of grains per spike, number of spikelets per plant, 1000grain weight, grain yield per plant, biological yield per plant, harvest index and protein content.

The data obtained were subjected to statistical analysis to get information on significance of differences and combining ability (Griffing B. et al., 1956 method 2, Model I).

The statistical model used for said analysis was:

$\mathrm{X}_{\mathrm{ij}}=\mu+\mathrm{g}_{\mathrm{i}}+\mathrm{g}_{\mathrm{j}}+\mathrm{s}_{\mathrm{ij}}+\frac{1}{r} \sum_{b} e_{i j} \quad\{\mathrm{i}, \mathrm{j}=1, \ldots \ldots . \mathrm{p}$ and $\mathrm{b}=1, \ldots . . \mathrm{r}$ \} 
Where,

$X_{\mathrm{ij}}=$ Mean value of experimental genotype,

$\mu=$ Population mean,

$\mathrm{g}_{\mathrm{i}}\left(\mathrm{g}_{\mathrm{j}}\right)=$ General combining ability (GCA) effect for $\mathrm{i}^{\text {th }}\left(\mathrm{j}^{\text {th }}\right)$ parent,

$\mathrm{s}_{\mathrm{ij}}=$ Specific combining ability (SCA) effect for cross between $i^{\text {th }}$ and $j^{\text {th }}$ parent assuming $s_{i j}$ $=\mathrm{s}_{\mathrm{ji}}$

$\mathrm{e}_{\mathrm{ijb}}=$ Error component pertaining to $\mathrm{i}^{\text {jth }}$ observation in $b^{\text {th }}$ replication,

$\mathrm{p}=$ Number of parents and

$\mathrm{r}=$ Number of replications.

\section{Results and Discussion}

The analysis of variance revealed that while, mean squares due to general combining ability (GCA) was significant for all the traits. Specific combining ability (SCA) effects were significant for all the traits, expect for days to 75 per cent maturity. Table 1

Parents RD 2715, BH 959, BH 393 can be utilizing for developing of a variety by exploiting additive gene action.

Perusal of Table 2 Out of 36 crosses, five crosses viz., RD $2786 \times \mathrm{BH} 959$ (21.07), BH $959 \times$ DWRB 64 (20.23), DWRB $91 \times$ BH 959 (20.22), RD $2035 \times$ RD 2786 (20.06) and RD $2715 \times$ BH 959 (19.97) exhibited higher per se performance for grain yield per plant.

3 crosses viz., DWRB $91 \times$ BH 959, RD 2035× RD 2786 and BH $959 \times$ DWRB 64 expressed higher significant sca effects for grain yield per plant. The significant specific combining ability effects (SCA) might be due to the occurrence of inter-allelic interaction and can be easily exploited in cross pollinated crops and in self-pollinated crops where commercial hybrid seed production is possible.

Parent BH 393 has been excellent general combiner for majority of traits viz., days to 50 per cent heading, days to 75 per cent maturity. Another parent BH 959 has good general combiner for plant height, flag leaf area, number of effective tillers per plant, spike length, number of grains per spike, number of spikelets per plant, 1000-grain weight, and grain yield per plant.

Followed by Parent RD 2786 has been good general combiner for protein content. BH 959 and DWRB 64 these both the parents are of higher yielding these both the parent of higher grain yielding crosses viz., RD $2786 \times \mathrm{BH}$ 959, RD $2552 \times$ DWRB 64, and RD $2035 \times$ RD 2786.

Among the parents, 4 parents expressed positive significant gca effects in positive direction with the magnitude varied from 0.56 (RD 2715) to 1.74 (BH 959). The positive significant sca effects for grain yield per plant were exhibited by 11 crosses with the magnitude ranged from 1.17 (DWRB $91 \times$ DWRB 64) to 2.50 (DWRB $91 \times$ BH 959). These results are in similar to the finding of Amer et al., (2012), Potla et al., (2013), Sultan et al., (2016), Rathore et al., (2017), Ram et al., (2017) and Patial et al., (2016).

Out of 36 crosses only two crosses viz., DWRB 91× BH 959 and RD $2552 \times$ DWRB 64 exhibited higher sca effects for grain yield per plant. As well as yield attributing traits, viz., number of grains per spike. with respect to protein content maximum sca effects for protein content were expressed by cross RD $2552 \times$ RD 2786 (1.20) followed by DWRB $91 \times$ BH 959 and RD $2035 \times$ RD 2786. Table $3-4$ 
Table.1 Combining ability mean square and EMS for thirteen characters in Barley (Hordeum vulgare L.)

\begin{tabular}{|c|c|c|c|c|c|c|}
\hline \multirow[t]{2}{*}{ SN. } & \multirow[t]{2}{*}{ Characters } & \multicolumn{3}{|c|}{ Source } & \multicolumn{2}{|c|}{ Var Model I } \\
\hline & & GCA & SCA & Error & GCA & SCA \\
\hline & & [8] & [36] & {$[88]$} & & \\
\hline 1 & Days to 50 per cent heading & $15.14 * *$ & $5.67 *$ & 3.27 & 8.63 & 86.28 \\
\hline 2 & Days to 75 per cent maturity & $22.44 * *$ & 6.17 & 6.07 & 11.90 & 3.37 \\
\hline 3 & Plant height $(\mathrm{cm})$ & $29.00 * *$ & $15.08 * *$ & 4.60 & 17.74 & 377.21 \\
\hline 4 & Flag leaf area $\left(\mathrm{cm}^{2}\right)$ & $55.58 * *$ & $8.85 * *$ & 0.38 & 40.14 & 304.76 \\
\hline 5 & $\begin{array}{c}\text { Number of effective tillers per } \\
\text { plant }\end{array}$ & $11.64 * *$ & $1.70 * *$ & 0.26 & 8.27 & 51.77 \\
\hline 6 & Spike length $(\mathrm{cm})$ & $6.94 * *$ & $2.78 * *$ & 0.55 & 4.65 & 80.31 \\
\hline 7 & Number of grains per spike & $1064.71 * *$ & $99.81 * *$ & 1.72 & 773.08 & 3531.40 \\
\hline 8 & Number of spikelets per plant & $4146.58 * *$ & $744.47 * *$ & 39.99 & 2986.61 & 25361.53 \\
\hline 9 & 1000-Grain weight $(\mathrm{g})$ & $11.40 * *$ & $2.15 * *$ & 1.02 & 7.56 & 40.74 \\
\hline 10 & Grain yield per plant (g) & $11.80 * *$ & $1.99 * *$ & 0.31 & 8.35 & 60.53 \\
\hline 11 & Biological yield per plant ( $\mathrm{g}$ ) & $10.74 *$ & $13.30 * *$ & 4.16 & 4.79 & 329.20 \\
\hline 12 & Harvest index \% & $14.82 * *$ & $6.79 * *$ & 1.61 & 9.61 & 186.33 \\
\hline 13 & Protein content $\%$ & $0.79 * *$ & $0.37 * *$ & 0.02 & 0.56 & 12.45 \\
\hline
\end{tabular}

$*, * *$ Significant at $5 \%$ and $1 \%$ level of significance, respectively.

Table.2 Identified on the basis of per se performance, for grain yield and protein content as well as sca/gca in Barley

\begin{tabular}{|c|c|c|c|c|}
\hline $\begin{array}{l}\text { S. } \\
\text { No. }\end{array}$ & Crosses & $\begin{array}{c}\text { Per se } \\
\text { performance } \\
\text { for grain } \\
\text { yield per } \\
\text { plant }(\mathrm{g})\end{array}$ & $\begin{array}{l}\text { Sca/gca } \\
\text { effects } \\
\text { for grain } \\
\text { yield per } \\
\text { plant }\end{array}$ & $\begin{array}{c}\text { Sca/gca } \\
\text { effects } \\
\text { for } \\
\text { protein } \\
\text { content }\end{array}$ \\
\hline 1. & RD $2786 \times$ BH 959 & 21.07 & 0.94 & $0.39 * *$ \\
\hline 2. & BH $959 \times$ DWRB 64 & 20.23 & $1.51 *$ & $-0.43 * *$ \\
\hline 3. & DWRB $91 \times$ BH959 & 20.22 & $2.50 * *$ & $1.14 * *$ \\
\hline 4. & RD $2035 \times$ RD 2786 & 20.06 & $1.97 * *$ & $1.13 * *$ \\
\hline 5. & RD $2715 \times$ BH 959 & 19.97 & 0.16 & $0.70 * *$ \\
\hline 6. & RD 2035 & 15.27 & -0.30 & -0.07 \\
\hline 7. & RD 2786 & 20.21 & $0.87 * *$ & -0.07 \\
\hline 8. & RD 2715 & 17.46 & $0.56 * *$ & 0.05 \\
\hline 9. & DWRB 91 & 13.73 & $-1.54 * *$ & $0.13 * *$ \\
\hline 10. & BH 959 & 19.94 & $1.74 * *$ & $0.59 * *$ \\
\hline 11. & DWRB 64 & 15.67 & $-0.54 * *$ & $-0.31 * *$ \\
\hline 7. & BH 946 & 19.36 & & \\
\hline
\end{tabular}

Best check- BH 946

$*$, ** Significant at $5 \%$ and $1 \%$ level of significance respectively. 
Table.3 GCA and SCA effects for Days to 50 per cent heading, Days to 75 per cent maturity, Plant height, Flag leaf area, Number of effective tillers per plant, Spike length and Number of grains per spike

\begin{tabular}{|c|c|c|c|c|c|c|c|c|}
\hline S N. & Genotype & $\begin{array}{l}\text { Days to } 50 \\
\text { per cent } \\
\text { heading }\end{array}$ & $\begin{array}{l}\text { Days to } 75 \\
\text { per cent } \\
\text { maturity }\end{array}$ & $\begin{array}{l}\text { Plant } \\
\text { height }\end{array}$ & $\begin{array}{c}\text { Flag leaf } \\
\text { area }\end{array}$ & $\begin{array}{l}\text { Number of } \\
\text { effective tillers } \\
\text { per plant }\end{array}$ & $\begin{array}{l}\text { Spike } \\
\text { length }\end{array}$ & $\begin{array}{c}\text { Number of } \\
\text { grains per } \\
\text { spike }\end{array}$ \\
\hline 1 & RD 2035 & -0.97 & 0.40 & $-1.38^{*}$ & $2.73 * *$ & $1.03 * *$ & $0.69 * *$ & $5.81 * *$ \\
\hline 2 & RD 2552 & 0.70 & $2.67 * *$ & 0.80 & $-3.19 * *$ & $-0.39 * *$ & -0.36 & $-1.69^{* *}$ \\
\hline 3 & RD 2786 & $-1.15 *$ & 0.58 & 0.72 & $-0.61 * *$ & $0.54 * *$ & $0.65^{* *}$ & $1.96^{* *}$ \\
\hline 4 & RD 2715 & $1.34 *$ & -1.18 & $2.89 * *$ & $1.74 * *$ & 0.19 & 0.24 & $5.05^{* *}$ \\
\hline 5 & DWRB 91 & -0.91 & $-1.72 *$ & -0.69 & $-1.56^{* *}$ & -0.18 & $-1.16^{* *}$ & $-12.54 * *$ \\
\hline 6 & ВН 959 & -0.21 & -1.36 & $-1.61 * *$ & $2.59 * *$ & $1.43 * *$ & $1.14^{* *}$ & $9.99 * *$ \\
\hline 7 & DWRB 92 & -0.21 & 1.07 & $1.68 * *$ & $-2.31 * *$ & $-1.85^{* *}$ & 0.11 & $-19.72 * *$ \\
\hline 8 & DWRB 64 & -0.81 & 0.55 & $-1.88^{* *}$ & $-1.24 * *$ & $0.36^{*}$ & $-1.13 * *$ & $5.34 * *$ \\
\hline 9 & BH 393 & $2.22 * *$ & -1.02 & -0.54 & $1.84 * *$ & $-1.14 * *$ & -0.18 & $5.81 * *$ \\
\hline 10 & RD $2035 \times$ RD 2552 & -0.70 & -3.67 & -1.46 & $-2.68 * *$ & $-1.36^{* *}$ & $1.44 *$ & -2.01 \\
\hline 11 & RD $2035 \times$ RD 2786 & -2.52 & -4.24 & $-4.59 *$ & $5.50 * *$ & $1.93 * *$ & $1.79 * *$ & $8.73 * *$ \\
\hline 12 & RD $2035 \times$ RD 2715 & 3.00 & 2.18 & $6.53 * *$ & $1.67 * *$ & $-2.05^{* *}$ & 0.58 & $5.44 * *$ \\
\hline 13 & RD $2035 \times$ DWRB 91 & -0.09 & 3.06 & -0.78 & $-3.36^{* *}$ & $-1.02 *$ & 0.10 & $-16.86^{* *}$ \\
\hline 14 & RD $2035 \times$ BH 959 & 1.55 & 1.36 & 2.80 & $-6.72 * *$ & $2.59 * *$ & -0.47 & $3.18 * *$ \\
\hline 15 & RD $2035 \times$ DWRB 92 & 2.55 & 0.27 & -2.72 & $-3.35 * *$ & $-1.65^{* *}$ & $-1.70 *$ & $-10.34 * *$ \\
\hline 16 & RD $2035 \times$ DWRB 64 & 2.48 & 2.79 & 0.81 & $2.05^{* *}$ & $-2.18^{* *}$ & $-1.43 *$ & $8.09 * *$ \\
\hline 17 & RD $2035 \times$ BH 393 & -2.21 & 1.03 & 0.22 & -0.82 & $-1.01^{*}$ & -0.08 & $-4.61 * *$ \\
\hline 18 & RD $2552 \times$ RD 2786 & -1.52 & 1.82 & 1.77 & $-1.19^{*}$ & -0.07 & -0.42 & 2.13 \\
\hline 19 & RD $2552 \times$ RD 2715 & $-3.33^{*}$ & -0.09 & 0.45 & $-3.04 * *$ & -0.65 & -0.91 & 2.01 \\
\hline 20 & RD $2552 \times$ DWRB 91 & -0.76 & 1.12 & 1.84 & -0.82 & $1.04 *$ & $-3.05 * *$ & $-8.50 * *$ \\
\hline 21 & RD $2552 \times$ BH 959 & 2.21 & 1.09 & 0.33 & $-1.93 * *$ & 0.11 & -0.54 & -0.59 \\
\hline 22 & RD $2552 \times$ DWRB 92 & $5.55 * *$ & -1.00 & $-6.41 * *$ & $4.53^{* *}$ & 0.38 & 1.28 & $-4.61 * *$ \\
\hline 23 & RD $2552 \times$ DWRB 64 & -0.18 & 1.52 & 0.45 & $-1.17 *$ & -0.42 & 0.61 & $4.42 * *$ \\
\hline 24 & RD $2552 \times$ BH 393 & 2.12 & 1.76 & -0.02 & $4.79 * *$ & 0.64 & -0.36 & 1.72 \\
\hline 25 & RD $2786 \times$ RD 2715 & -1.15 & -0.33 & 1.80 & -0.76 & -0.19 & 1.06 & 2.29 \\
\hline 26 & RD $2786 \times$ DWRB 91 & 1.42 & 1.21 & 0.22 & $-3.44 * *$ & 0.55 & $-2.51 * *$ & $-9.38 * *$ \\
\hline 27 & RD $2786 \times$ BH 959 & $3.73 *$ & -2.82 & 1.02 & $4.83^{* *}$ & -0.60 & $2.81 * *$ & -1.01 \\
\hline 28 & RD $2786 \times$ DWRB 92 & -0.61 & 0.76 & -2.07 & 0.21 & -0.43 & $-2.33 * *$ & $-4.00 * *$ \\
\hline 29 & RD $2786 \times$ DWRB 64 & 0.33 & -1.39 & 2.27 & $2.18^{* *}$ & 0.15 & $-1.93 * *$ & $-3.33 * *$ \\
\hline 30 & RD $2786 \times$ BH 393 & -1.03 & $4.52 *$ & -1.81 & $-3.68 * *$ & 0.66 & -0.81 & -1.73 \\
\hline 31 & RD $2715 \times$ DWRB 91 & $4.27 *$ & 1.30 & -1.29 & -1.10 & 0.27 & $-1.61 *$ & $-19.44 * *$ \\
\hline 32 & RD 2715 × BH 959 & -0.76 & -1.73 & $-7.23^{* *}$ & $2.12 * *$ & $2.66 * *$ & 0.68 & $6.80^{* *}$ \\
\hline 33 & RD $2715 \times$ DWRB 92 & -0.76 & -0.82 & 1.28 & $-1.20^{*}$ & -0.80 & -0.03 & $-8.06^{* *}$ \\
\hline 34 & RD $2715 \times$ DWRB 64 & -1.48 & -4.30 & -2.10 & -0.85 & 0.87 & -0.93 & $-5.49 * *$ \\
\hline 35 & RD $2715 \times$ BH 393 & 0.15 & 2.94 & 1.36 & -1.09 & -0.08 & -0.19 & $-3.82 * *$ \\
\hline 36 & DWRB 91 × BH 959 & $-4.18 *$ & -2.85 & $-5.57 * *$ & $4.81 * *$ & $1.06^{*}$ & $2.09 * *$ & $23.26^{* *}$ \\
\hline 37 & DWRB $91 \times$ DWRB 92 & 0.15 & 2.06 & $6.90 * *$ & -0.85 & -0.02 & $2.84 * *$ & $5.21 * *$ \\
\hline 38 & DWRB $91 \times$ DWRB 64 & -1.24 & -2.09 & 0.47 & 0.18 & -0.67 & $1.76^{*}$ & $13.18 * *$ \\
\hline 39 & DWRB $91 \times$ BH 393 & 2.73 & -0.85 & -0.05 & -0.92 & -0.53 & 0.67 & $10.74 * *$ \\
\hline 40 & BH $959 \times$ DWRB 92 & -1.21 & 4.03 & $4.33^{*}$ & 1.01 & $-1.58^{* *}$ & -0.93 & $-16.22 * *$ \\
\hline 41 & BH $959 \times$ DWRB 64 & 0.39 & 0.88 & $-5.37 * *$ & $-4.15 * *$ & $-0.94 *$ & $-4.15 * *$ & $-14.29 * *$ \\
\hline 42 & BH $959 \times$ BH 393 & -0.64 & -0.21 & 2.71 & -1.04 & -0.37 & -0.17 & $-2.59 *$ \\
\hline 43 & DWRB $64 \times$ DWRB 92 & 0.06 & 2.12 & $11.45^{* *}$ & -0.10 & $2.81 * *$ & $2.38 * *$ & $-8.17 * *$ \\
\hline 44 & DWRB $92 \times$ BH 393 & $-3.97 *$ & $-4.64 *$ & -1.72 & $1.96^{* *}$ & -0.01 & 0.43 & $20.09 * *$ \\
\hline \multirow[t]{8}{*}{45} & DWRB $64 \times$ BH 393 & -3.03 & 2.55 & 0.53 & $1.56^{* * *}$ & 0.59 & $1.53 *$ & $-3.94 * *$ \\
\hline & \multicolumn{2}{|l|}{ Standard error } & & & & & & \\
\hline & $\mathrm{Gi}$ & 0.51 & 0.70 & 0.61 & 0.18 & 0.14 & 0.21 & 0.37 \\
\hline & $\mathrm{Gi}-\mathrm{Gj}$ & 0.77 & 1.05 & 0.91 & 0.26 & 0.22 & 0.32 & 0.56 \\
\hline & Sii & 1.46 & 1.99 & 1.74 & 0.50 & 0.41 & 0.60 & 1.06 \\
\hline & Sij & 1.65 & 2.25 & 1.96 & 0.57 & 0.46 & 0.68 & 1.20 \\
\hline & Sij-ik & 2.44 & 3.32 & 2.89 & 0.84 & 0.69 & 1.00 & 1.77 \\
\hline & Sij-Skl & 2.31 & 3.15 & 2.74 & 0.79 & 0.65 & 0.95 & 1.68 \\
\hline
\end{tabular}

$*$, ** Significant at $5 \%$ and $1 \%$ level of significance respectively. 
Table.4 GCA and SCA effects for Number of spikelets per plant, 1000-Grain weight, Grain yield per plant, Biological yield per plant, Harvest index, and Protein content

\begin{tabular}{|c|c|c|c|c|c|c|c|}
\hline SN. & Genotype & $\begin{array}{l}\text { Number of } \\
\text { spikelets } \\
\text { per plant }\end{array}$ & $\begin{array}{c}\text { 1000-Grain } \\
\text { weight }\end{array}$ & $\begin{array}{c}\text { Grain yield per } \\
\text { plant }\end{array}$ & $\begin{array}{c}\text { Biological } \\
\text { yield per } \\
\text { plant }\end{array}$ & $\begin{array}{c}\text { Harvest } \\
\text { index }\end{array}$ & $\begin{array}{l}\text { Protein } \\
\text { content }\end{array}$ \\
\hline 1 & RD 2035 & $22.22 * *$ & 0.15 & -0.30 & -0.76 & 0.10 & -0.07 \\
\hline 2 & RD 2552 & $-15.05 * *$ & $-0.90^{* *}$ & $-0.56^{* *}$ & 0.53 & $-1.09^{* *}$ & 0.07 \\
\hline 3 & RD 2786 & $8.88 * *$ & $1.06^{* *}$ & $0.87 * *$ & 0.86 & 0.48 & -0.07 \\
\hline 4 & RD 2715 & $5.04 * *$ & -0.14 & $0.56^{* * *}$ & 0.54 & 0.60 & 0.05 \\
\hline 5 & DWRB 91 & $4.57^{*}$ & $-1.61 * *$ & $-1.54 * *$ & -0.60 & $-1.72 * *$ & $0.13^{* *}$ \\
\hline 6 & ВН 959 & $25.83 * *$ & $1.60^{* *}$ & $1.74 * *$ & -0.05 & $2.20^{* *}$ & $0.59^{* *}$ \\
\hline 7 & DWRB 92 & $-23.29 * *$ & $-0.81 * *$ & $-0.95^{* * *}$ & -0.45 & $-1.03^{* *}$ & $-0.31 * *$ \\
\hline 8 & DWRB 64 & 2.68 & -0.05 & $-0.54 * *$ & $-1.68 * *$ & 0.32 & $-0.31 * *$ \\
\hline 9 & BH 393 & $-30.88 * *$ & $0.69 *$ & $0.72 * *$ & $1.59 * *$ & 0.13 & -0.07 \\
\hline 10 & RD $2035 \times$ RD 2552 & $-24.03 * *$ & 0.30 & -0.87 & -0.23 & -1.02 & $-0.46^{* *}$ \\
\hline 11 & RD $2035 \times$ RD 2786 & $59.84 * *$ & -0.21 & $1.97 * *$ & -1.49 & $3.18^{* *}$ & $1.13^{* *}$ \\
\hline 12 & RD $2035 \times$ RD 2715 & $-41.37 * *$ & -0.44 & -0.82 & 0.82 & -1.78 & -0.13 \\
\hline 13 & RD $2035 \times$ DWRB 91 & $-18.81 * *$ & 1.39 & 0.51 & -1.31 & 1.32 & $0.28^{*}$ \\
\hline 14 & RD $2035 \times$ BH 959 & $38.65^{* *}$ & 1.44 & 0.11 & 2.13 & -1.21 & -0.24 \\
\hline 15 & RD $2035 \times$ DWRB 92 & $-27.89 * *$ & -0.02 & $1.22 *$ & 2.50 & 0.15 & $0.77 * *$ \\
\hline 16 & RD $2035 \times$ DWRB 64 & $-44.07 * *$ & $2.06^{*}$ & -0.57 & 0.21 & -0.85 & $0.32 *$ \\
\hline 17 & RD $2035 \times$ BH 393 & $-28.54 * *$ & -0.86 & $1.75 * *$ & $10.03 * *$ & $-2.89 *$ & -0.00 \\
\hline 18 & RD $2552 \times$ RD 2786 & $26.51 * *$ & -0.86 & -0.44 & -0.44 & -0.04 & $1.20^{* *}$ \\
\hline 19 & RD $2552 \times$ RD 2715 & $-25.79 * *$ & $1.85^{*}$ & $1.30^{*}$ & 0.40 & 1.17 & -0.02 \\
\hline 20 & RD $2552 \times$ DWRB 91 & $20.54 * *$ & 1.23 & $-1.96^{* * *}$ & -1.12 & -1.99 & 0.16 \\
\hline 21 & RD $2552 \times$ BH 959 & 3.71 & -1.53 & -0.52 & 1.28 & -1.32 & $-0.89 * *$ \\
\hline 22 & RD $2552 \times$ DWRB 92 & -10.37 & 1.70 & 0.26 & 0.37 & 0.25 & $-0.78^{* *}$ \\
\hline 23 & RD $2552 \times$ DWRB 64 & $11.64 *$ & 1.18 & $1.98 * *$ & 2.95 & 0.79 & 0.07 \\
\hline 24 & RD $2552 \times$ BH 393 & 4.45 & -1.51 & -0.05 & -1.96 & 0.98 & 0.24 \\
\hline 25 & RD $2786 \times$ RD 2715 & $-17.11 * *$ & -0.58 & -0.40 & -0.07 & -0.64 & $-0.91 * *$ \\
\hline 26 & RD $2786 \times$ DWRB 91 & -0.18 & $-2.34 *$ & $-1.42^{* * *}$ & -0.04 & -1.56 & $-0.38 * *$ \\
\hline 27 & RD $2786 \times$ BH 959 & $-25.82 * *$ & $2.69 * *$ & 0.94 & 3.20 & -0.80 & $0.39 * *$ \\
\hline 28 & RD $2786 \times$ DWRB 92 & $12.17^{*}$ & -0.25 & 0.81 & -1.56 & 2.08 & -0.21 \\
\hline 29 & RD $2786 \times$ DWRB 64 & $-12.28^{*}$ & -1.39 & $-3.42^{* *}$ & 1.10 & $-4.92 * *$ & 0.15 \\
\hline 30 & RD $2786 \times$ BH 393 & $25.00^{* *}$ & -1.46 & 0.07 & -0.24 & 0.16 & $-0.52 * *$ \\
\hline 31 & RD $2715 \times$ DWRB 91 & $15.29 * *$ & -1.51 & $1.54 * *$ & 0.12 & 1.70 & 0.15 \\
\hline 32 & RD $2715 \times$ BH 959 & $60.07 * *$ & 0.94 & 0.16 & $-13.72 * *$ & $9.68 * *$ & $0.70^{* *}$ \\
\hline 33 & RD $2715 \times$ DWRB 92 & 8.36 & -1.81 & -0.69 & -0.12 & -0.98 & $-0.69 * *$ \\
\hline 34 & RD $2715 \times$ DWRB 64 & 2.89 & -0.51 & $1.65 * *$ & 2.40 & 0.33 & -0.15 \\
\hline 35 & RD $2715 \times$ BH 393 & -4.36 & -0.03 & -0.41 & 2.29 & -1.95 & $-0.64 * *$ \\
\hline 36 & DWRB $91 \times$ BH 959 & $29.44 * *$ & 1.79 & $2.50 * *$ & 0.49 & $2.72 *$ & $1.14 * *$ \\
\hline 37 & DWRB $91 \times$ DWRB 92 & $-11.74 *$ & 0.97 & 0.71 & 0.06 & 0.91 & 0.18 \\
\hline 38 & DWRB $91 \times$ DWRB 64 & -7.82 & -0.44 & $1.17 *$ & 1.00 & 0.94 & $-0.37 *$ \\
\hline 39 & DWRB $91 \times$ BH 393 & -11.48 & 0.85 & $-1.64^{* * *}$ & -1.62 & -1.21 & 0.08 \\
\hline 40 & BH $959 \times$ DWRB 92 & $-24.83 * *$ & 0.95 & $-1.92 * *$ & -0.71 & -2.03 & $0.60 * *$ \\
\hline 41 & BH $959 \times$ DWRB 64 & $-15.53 * *$ & -1.44 & $1.51^{* * *}$ & 2.61 & 0.04 & $-0.43^{* *}$ \\
\hline 42 & BH $959 \times$ BH 393 & -0.72 & -1.10 & -0.67 & 0.00 & -1.15 & 0.18 \\
\hline 43 & DWRB $64 \times$ DWRB 92 & $27.58^{* *}$ & 0.11 & 0.38 & 0.79 & -0.03 & 0.28 \\
\hline 44 & DWRB $92 \times$ BH 393 & -6.37 & 0.67 & $1.67 * *$ & -0.72 & 2.30 & $0.56^{* *}$ \\
\hline \multirow[t]{8}{*}{45} & DWRB $64 \times$ BH 393 & $16.04 * *$ & -0.15 & $-1.15^{*}$ & 1.16 & -2.27 & -0.13 \\
\hline & Standard error & & & & & & \\
\hline & $\mathrm{Gi}$ & 1.80 & 0.29 & 0.16 & 0.58 & 0.36 & 0.04 \\
\hline & $\mathrm{Gi}-\mathrm{Gj}$ & 2.70 & 0.43 & 0.24 & 0.87 & 0.54 & 0.07 \\
\hline & Sii & 5.12 & 0.82 & 0.45 & 1.65 & 1.03 & 0.13 \\
\hline & $\mathrm{Sij}$ & 5.78 & 0.92 & 0.51 & 1.87 & 1.16 & 0.14 \\
\hline & Sij-ik & 8.53 & 1.36 & 0.75 & 2.75 & 1.71 & 0.21 \\
\hline & Sij-Skl & 8.09 & 1.29 & 0.71 & 2.61 & 1.62 & 0.20 \\
\hline
\end{tabular}

$*$, ** Significant at $5 \%$ and $1 \%$ level of significance respectively. 
The study under presents were revealed that some of the parents viz., BH 959 and RD 2786 used in present analysis can be preferred for the successful development of variety through pedigree method.

Parents were noted as good sources of encouraging genes for increasing grain yield and exploit of these parents would be more gratifying for boosting grain yield in Barley.

A perusal of specific combining ability effects revealed that positive significant sca effects for grain yield per plant was observed in eleven crosses viz., DWRB $91 \times$ BH 959, RD $2552 \times$ DWRB 64, RD $2035 \times$ RD 2786, RD $2035 \times$ BH 393, DWRB $92 \times$ BH 393 RD $2715 \times$ DWRB 64, RD $2715 \times$ DWRB 91, BH $959 \times$ DWRB 64, RD $2552 \times$ RD 2715, RD $2035 \times$ DWRB 92 and DWRB $91 \times$ DWRB 64 expressed higher positive significant sca effects ranged from 1.17 to 2.50 along with good per se performance from 14.44 to 21.07 .

Table 4 In general a close association between sca effects and per se performance for grain yields per plant and protein content was recorded amongst the five promising crosses.

These five crosses appear to be very capable combination and could be recommended for multi-location trial.

\section{References}

Amer, Kh, A Eid, A., Elakhdar, A. and ElShawy, E. 2012. Combining ability and heterosis in five barley genotypes for some economic traits. Egypt Journal Agriculture Research, 90: 105-116.

Baik, B.K and Ullrich, SE. 2008. Barley for food characteristics, improvement and renewed interest. Journal of cereal science, 48:233-242

Griffing, B. (1956a). Concepts of specific and general combining ability in relation to diallel crossing systems. Australian Journal Biol Science, 9:463-493.

Koulagi, R., Kaur S. Bishnoi SP. and Kanwar RS. 2018. Identification of resistance source to cereal cyst nematode, Heterodera avenae wollenweber in barley (Hordeum vulgare L.) Wheat and Barley Research, 10(3): 231-235

Patial, M., Pal, D. and Kumar, J. 2016. Combining ability and gene action studies for grain yield and its component traits in Barley (Hordeum vulgare L.) SABRAO Journal of Breeding and Genetics 48 (1) 90-96.

Potla, K.R., Bornare, S. S., Prasad, L.C., Prasad, R. and Madakemohekar, A.H. 2013. Study of heterosis and combining ability for yield and yield contributing traits in barley (Hordeum vulgare L.) The Bioscan, 8(4): 1231-1235.

Ram, M. and Shekhawat, A.S. 2017. Heterosis, inbreeding depression and combining ability analysis for yield and its component traits in barley (Hordeum vulgare L.). Plant Archives 17(2): 851860.

Rathore, R. K. S. and Chauhan, Y. 2017. GCA and SCA effects analysis for grain yield and its quantitative traits in sixrowed barley (Hordeum vulgare L.) in agra region. Indian Journal Sciences Research, 16(1): 56-63.

Sultan, M. S., Abdel-Moneam, M. A. and Haffez, S. H. 2016. Estimation of combining ability for yield and its components in barley under normal and stress drought condition. Journal Plant Production, Mansoura University, 7(6): $553-558$. 


\section{How to cite this article:}

Deepak Panwar and Hemlata Sharma. 2019. Study of Combining Ability Analysis in Barley (Hordeum vulgare L.). Int.J.Curr.Microbiol.App.Sci. 8(12): 3004-3011.

doi: https://doi.org/10.20546/ijcmas.2019.812.349 